\title{
Neurofibroma plexiforme de punta nasal
}

\section{Plexiform neurofibromatosis of nasal TIP}

\author{
Sergio Pérez B¹, Cecilia Varela U², Marcelo Del Villar U.
}

\begin{abstract}
RESUMEN
La neurofibromatosis es una enfermedad genética autosómica dominante y de alta penetrancia que pertenece al grupo de los síndromes neurocutáneos. Se sabe de diversas variedades de neurofibromatosis; sin embargo, las más conocidas son la neurofibromatosis tipo 1 (enfermedad de von Recklinghausen) y neurofibromatosis tipo 2 (neurofibromatosis del nervio acústico bilateral).

Se presenta el caso clínico de un paciente de 43 años, sexo femenino, sin antecedentes familiares de importancia, quien presentó aumento de volumen de punta nasal, de lenta progresión, asociado a obstrucción nasal y anosmia significativa. Al examen físico destacaban múltiples manchas de color 'café con leche' y nódulos de diferentes tamaños distribuidos en tórax.

El estudio imagenológico con tomografía computarizada de cavidades paranasales mostró un nódulo de partes blandas en la porción cartilaginosa del tabique nasal. Se le realizó una rinoplastía abierta con extirpación total del tumor, con diagnóstico histopatológico compatible de neurofibroma plexiforme de punta nasal.

Se analiza el caso del neurofibroma plexiforme de punta nasal, debido a la ubicación infrecuente de éste, y se efectúa una breve revisión bibliográfica del tema.
\end{abstract}

Palabras clave: Neurofibromatosis, enfermedad von Recklinghausen, neurofibroma plexiforme.

\section{ABSTRACT}

Neurofibromatosis is a genetic, autosomal dominant disease with high penetrance that belongs to the neurocutaneous syndrome group. There are several varieties of neurofibromatosis, of which the best known are neurofibromatosis type 1 (von Recklinghausen disease) and neurofibromatosis type 2 (schwannoma of the bilateral acoustic nerve).

We present a case report of a 43 years old female patient, with no relevant family medical history, whose nasal tip size slowly enlarged, in association with considerable nasal obstruction and anosmia. The physical examination highlighted multiple brown, coffee and milk colored spots and nodules of different sizes distributed in the chest and the back. The imaging study of paranasal cavities with computerized tomography showed a soft tissue nodule in the cartilaginous portion of the nasal septum. A total tumor resection and open rhinoplasty were performed. The histopatological diagnosis was plexiform neurofibroma of the nasal tip. We present the case given the unusual location of it and make a brief literature review of the topic.

Key word: Neurofibromatosis, von Recklinghausen disease, plexiform neurofibroma

1 Médico del Servicio de Otorrinolaringología del Hospital Antonio Tirado Lanas, Ovalle.

2 Escuela de Medicina, Universidad Diego Portales 


\section{INTRODUCCIÓN}

Los síndromes neurocutáneos son un conjunto de desórdenes genéticos que producen una serie de anormalidades, tanto en la piel como en el sistema nervios $0^{1}$. Dentro de este grupo se encuentra la neurofibromatosis, enfermedad genética autosómica dominante, de alta penetrancia y de curso progresivo ${ }^{2}$. Se han descrito diversas variedades; sin embargo, las más conocidas son la neurofibromatosis tipo I (NF-1), que representa el 85\%$90 \%$ de los casos y la neurofibromatosis tipo II (NF-2), muy poco frecuente ${ }^{3}$.

La NF-1 tiene una incidencia de 1:3.500 personas, sin predominio por sexo². Clínicamente se manifiesta con neurofibromas múltiples, cutáneos o plexiformes, manchas de color 'café con leche' y nódulos de Lisch, entre otros signos ${ }^{3}$.

Los neurofibromas se ubican frecuentemente en la piel y tejido subcutáneo. En cambio, los neurofibromas ubicados en el tracto respiratorio alto son infrecuentes, especialmente en cavidad nasal y senos paranasales ${ }^{4}$.

Actualmente existen pocos casos de neurofibroma de punta nasal publicados en la literatura. Por lo tanto, el propósito de este trabajo es presentar un caso clínico de neurofibroma plexiforme de punta nasal, debido a la ubicación infrecuente de éste, complementado con una breve revisión bibliográfica del tema.

\section{CASO CLÍnICO}

Paciente de sexo femenino, de 43 años, con antecedentes de malformación congénita de antebrazo izquierdo, sin datos familiares de importancia, quien consultó el año 2005 al Servicio de Otorrinolaringología por presentar un cuadro iniciado a los 12 años de edad, caracterizado por aumento de volumen en punta nasal, de lenta progresión e indoloro, asociado a obstrucción nasal significativa, anosmia y sin otra sintomatología de importancia.

Al examen físico, en la piel destacaban numerosas manchas de color 'café con leche' de diferentes tamaños, distribuidas en tórax, la mayor aproximadamente de $2 \mathrm{~cm}$ y, además, muchos nódulos en tórax y cuello de diversas dimensiones, el mayor de $4 \mathrm{~cm}$, de aspecto móvil, indoloro y no adherido a planos profundos (Figura 1).
Al examen físico dirigido se apreciaba un aumento de volumen en punta nasal de consistencia blanda, inmóvil, adherido a planos profundos, que obstruía de manera significativa ambas narinas (Figura 2).

Se le realizó tomografía computarizada de cavidades perinasales, la que mostró un nódulo de partes blandas en la porción cartilaginosa del tabique nasal e hipoplasia de los senos frontales (Figura 3).

Intervenida quirúrgicamente, se le realizó una extirpación total del tumor nasal vía rinoplastía abierta tenía un aspecto fibroso y muy vascularizado- junto con extracción de cartílago del tabique nasal para reconstrucción de la columela y punta nasal (Figura 4).

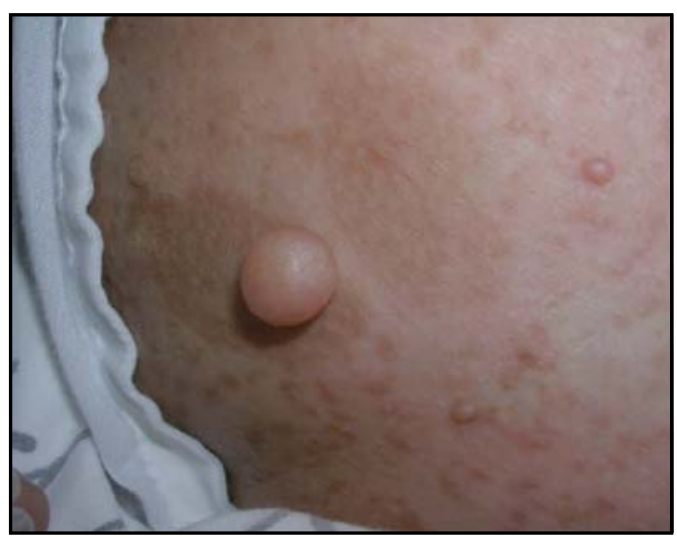

Figura 1. Múltiples nódulos y manchas color 'café con leche' en tórax.

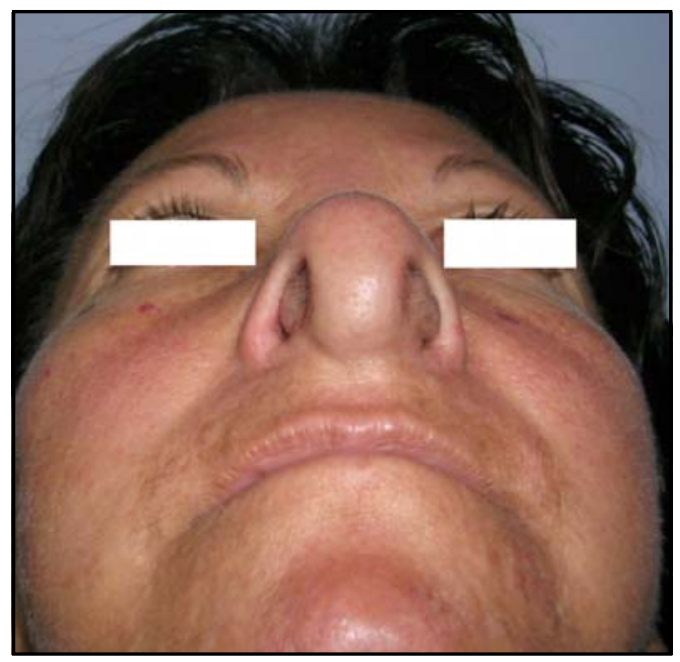

Figura 2. Obstrucción de narinas por tumoración en punta nasal. 


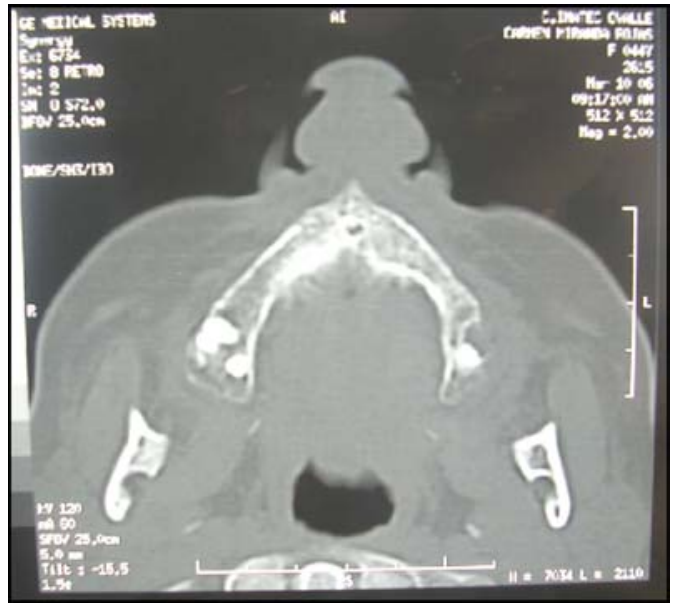

Figura 3. TC (corte axial): nódulo de partes blandas en septum cartilaginoso.

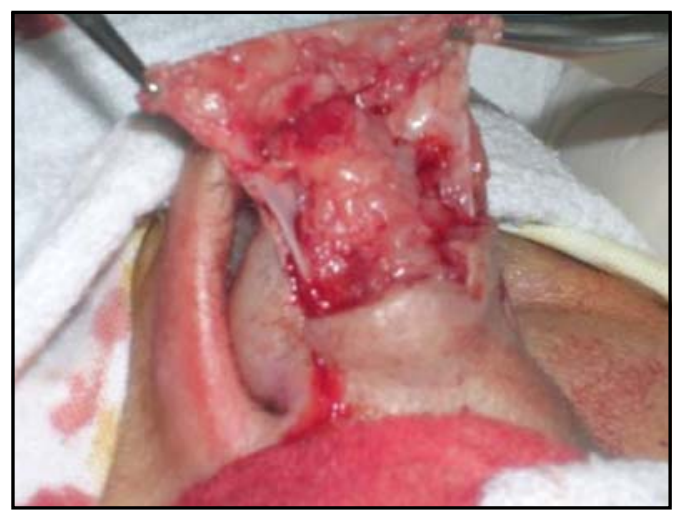

Figura 4. Tumor de aspecto fibroso y muy vascularizado.

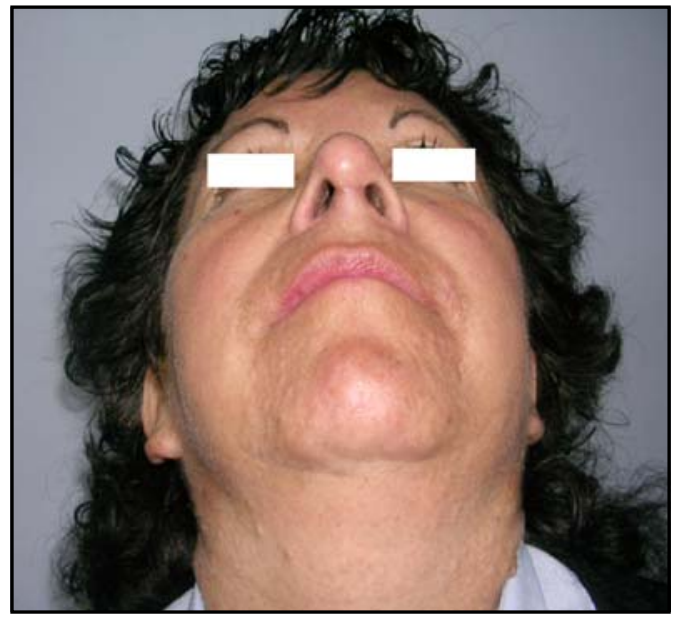

Figura 5. Paciente luego de un año de operada.
El estudio anátomo-patológico evidenció lesión tumoral compuesta por células de núcleos fusados y ovoideos, de citoplasma eosinofílico con borde citoplasmático poco nítido. La inmunohistoquímica fue compatible con S-100 (+), confirmando el diagnóstico de neurofibroma plexiforme de punta nasal.

La evolución posoperatoria fue satisfactoria, sin complicaciones y en buenas condiciones generales.

Actualmente la paciente se mantiene en control anual y, hasta la fecha, no se ha detectado recidiva (Figura 5).

\section{DISCUSIÓN}

La NF-1, también llamada enfermedad de von Recklinghausen, fue descrita por primera vez por Friedrich von Recklinghausen en $1882^{5}$. Esta enfermedad presenta una mutación en un gen supresor de tumor ubicado en el cromosoma 17. Se estima que la NF-1 tiene una incidencia de 1:3.500 personas al año, sin predominio de sexo, grupo étnico ni localización geográfica².

Clínicamente se manifiesta por la presencia de neurofibromas múltiples, cutáneos o plexiformes, manchas de color 'café con leche' y nódulos de Lisch. También se observan casos de pseudoartrosis, feocromocitoma, gliomas ópticos, anomalías óseas, retraso mental y dificultades para el aprendizaje ${ }^{3}$.

La primera manifestación clínica de la enfermedad corresponde a la aparición de manchas de color 'café con leche', presente en el $90 \%$ de los casos. Se distribuyen en forma aleatoria por el cuerpo y van creciendo con la edad 3 .

La localización más frecuente de los neurofibromas es piel y tejido subcutáneo. En cambio, los neurofibromas de cavidades nasales, senos paranasales y nasofaringe son infrecuentes ${ }^{6}$. Se han descrito pocos casos de neurofibromas ubicados en el antro maxilar, cavidad nasal, septum nasal, cornete medio y en glándulas salivales, entre otros ${ }^{4-9}$.

Dentro de la sintomatología más característica de los neurofibromas de cavidad nasal se describe epistaxis, obstrucción nasal, rinorrea mucopurulenta, hiposmia y dolor ${ }^{6}$. 
Tabla 1. Criterios diagnósticos de la neurofibromatosis tipo 1

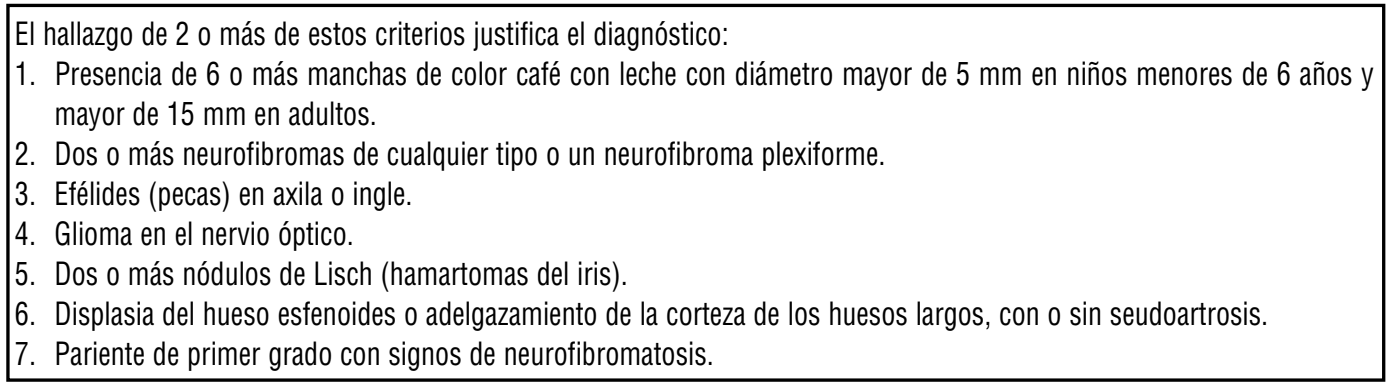

De: Gutmann DH et al. JAMA 1997; 278: 51-7.

El diagnóstico de NF-1 se realiza en base a criterios, descritos por el Instituto Nacional de Salud (NIH) de U.S.A., actualizado en el año $1997^{10}$ (Tabla 1). Éste se ratifica en base a la clínica, estudio imagenológico y examen anatomopatológico.

Dentro de los diagnósticos diferenciales de tumores nasales se incluye: linfoma, papiloma, angiofibroma juvenil, meningioma, schwanoma, encondroma, condrosarcoma y carcinoma escamoso de nariz.

Es importante el reconocimiento precoz de la enfermedad ya que la edad de comienzo se sitúa, en el $80 \%$ de los casos, antes de los 21 años $^{3}$.

Finalmente, es fundamental el seguimiento clínico a largo plazo de estos pacientes debido a que, en el $10 \%$ de los casos, los neurofibromas pueden malignizarse y convertirse en neurofibrosarcomas. Sin embargo, la recurrencia de estos tumores es poco frecuente ${ }^{6}$.

\section{BIBLIOGRAFÍA}

1. Sagar S, Israel M. Primary and metastatic tumors of the nervous system. En: Fauci A, ed. Harrison's Principles of Internal Medicine. $16^{\text {th }}$ ed. U.S.A.: McGraw-Hill, 2005; 2461-81.

2. Ars E, Kruyer H, Morell M, Pros M, Serra M, Ravella A, et al. Recurrent mutations in the NF1 gene are common among neurofibromatosis type 1 patients. J Med Genet 2003; 40: 1-8.

3. Estrella V, Habermacher S, Fernández R. Neurofibromatosis. Rev Med Rosario 2008; 74: 21-5.

4. Annino DJ JR, Domanowski GF, Vaughan CW. A rare cause of nasal obstruction: A solitary neurofibroma. Otolaryngol Head Neck Surg 1991; 104: 484-8.

5. Inzunza F, Celedón C, Olavarría C, Medrano M. Neurofibromatosis de glándulas salivales. Rev Otorrinolaringol Cir Cabeza Cuello 2003; 63: 49-54.

6. Fraczek M, Zalesska-Krecicka M, Zatonski T, KRECICKI T. Two cases of nasal neurofibroma. $J$ Otolaryngol 2006; 5: 1-9.

7. Agarwal MK, Gupta OP, Samant hC, Grupta S. Neurofibroma of the maxillary antrum. J Oral Surg 1979; 48: 150-2.

8. Hirao M, Gushiken T, Imokawa $\mathrm{H}$, Kawal $\mathrm{S}$, Inaba $\mathrm{H}$, TSUKUDA M. Solitary neurofibroma of the nasal cavity: Resection with endoscopic surgery. $J$ Laryngol Otol 2001; 115: 1012-4.

9. Yong DK, Chang HB, Jang SS, Kel WS. Transnasal endoscopic excision of an isolated neurofibroma of the nasal septum. Rhinology 1997; 35: 89-91.

10. Gutmann DH, Aylsworth A, Carey JC, et al. The diagnostic evaluation and multidisciplinary management of neurofibromatosis 1 and neurofibromatosis 2. JAMA 1997; 278: 51-7. 